\title{
The 5S rDNA in two Abracris grasshoppers (Ommatolampidinae: Acrididae): molecular and chromosomal organization
}

\author{
Danilo Bueno ${ }^{1}$ - Octavio Manuel Palacios-Gimenez ${ }^{1}$ Dardo Andrea Martí ${ }^{2}$. \\ Tatiane Casagrande Mariguela ${ }^{3} \cdot$ Diogo Cavalcanti Cabral-de-Mello $^{1}$
}

Received: 4 January 2016 / Accepted: 30 March 2016 / Published online: 22 April 2016

(C) Springer-Verlag Berlin Heidelberg 2016

\begin{abstract}
The 5S ribosomal DNA (rDNA) sequences are subject of dynamic evolution at chromosomal and molecular levels, evolving through concerted and/or birth-anddeath fashion. Among grasshoppers, the chromosomal location for this sequence was established for some species, but little molecular information was obtained to infer evolutionary patterns. Here, we integrated data from chromosomal and nucleotide sequence analysis for 5S rDNA in two Abracris species aiming to identify evolutionary dynamics. For both species, two arrays were identified, a larger sequence (named type-I) that consisted of the entire 5S rDNA gene plus NTS (non-transcribed spacer) and a smaller (named type-II) with truncated 5S rDNA gene plus short NTS that was considered a pseudogene. For typeI sequences, the gene corresponding region contained the internal control region and poly-T motif and the NTS presented partial transposable elements. Between the species, nucleotide differences for type-I were noticed, while typeII was identical, suggesting pseudogenization in a common ancestor. At chromosomal point to view, the type-II was
\end{abstract}

Communicated by S. Hohmann.

D. Bueno and O. M. Palacios-Gimenez equally contributed.

Diogo Cavalcanti Cabral-de-Mello

mellodc@rc.unesp.br

1 Departamento de Biologia, Instituto de Biociências/IB, UNESP, Univ Estadual Paulista, Rio Claro, São Paulo CEP 13506-900, Brazil

2 Laboratorio de Genética Evolutiva, IBS, Facultad de Ciencias Exactas, Químicas y Naturales, CONICET, Universidad Nacional de Misiones, 3300 Posadas, Argentina

3 Departamento de Zoologia, Instituto de Biociências/IB, UNESP, Univ Estadual Paulista, Rio Claro, São Paulo, Brazil placed in one bivalent, while type-I occurred in multiple copies in distinct chromosomes. In Abracris, the evolution of 5S rDNA was apparently influenced by the chromosomal distribution of clusters (single or multiple location), resulting in a mixed mechanism integrating concerted and birth-and-death evolution depending on the unit.

Keywords FISH $\cdot$ Genome $\cdot$ Multigene family $\cdot$ Nontranscribed spacer $\cdot$ Repetitive DNAs

\section{Introduction}

A multigene family is a group of multiple genes descending from a common ancestor presenting similar sequences and functional roles (Nei and Rooney 2005). The ribosomal RNA (rRNA) genes in higher Eukaryotes are represented by two distinct multigene families constituted of hundreds to thousands copies tandemly arrayed occupying one or more chromosomal loci. The $45 \mathrm{~S}$ ribosomal DNA (rDNA) comprises three distinct genes separated from each other by the internal transcribed spacer (ITS), coding for the $18 \mathrm{~S}$, 5.8S, and 28S rRNAs. The 5S rDNA is formed by multiple repeats of a highly conserved 120-bp sequence separated from each other by a non-transcribed spacer called the NTS that is subject to rapid evolution and presents variability in size and nucleotide composition (reviewed by Long and Dawid 1980). Based on the similarity between the $5 \mathrm{~S}$ rDNA sequences, some studies propose that this multigene family is subject to concerted evolution, a model in which multiple copies of a sequence are homogenized (Ney and Rooney 2005). On the contrary, other studies have proposed that in some genomes, the 5S rDNA evolves through birth-and-death evolution or in a mixed effect of concerted and birth-and-death evolution (see, e.g., Rooney and Ward 
2005; Úbeda-Manzanaro et al. 2010; Perina et al. 2011; Pinhal et al. 2011; Vierna et al. 2011, 2013; Vizoso et al. 2011; Merlo et al. 2013).

Cytogenetic or molecular characterization aiming at understanding 5S rDNA organization and evolution has been undertaken, e.g., in fish (Martins and Galetti Jr 2001; Wasko et al. 2001; Martins et al. 2002; Rebordinos et al. 2013), amphibians (Rodrigues et al. 2012), crustaceans (Perina et al. 2011), and mollusks (Vierna et al. 2011). These studies determined that the 5S rDNA and its NTS are good markers for species identification, phylogenetic analyses, karyotype reconstruction, and understanding genome evolution. A high variability in the number of chromosomal clusters has been noticed for 5S rDNA with a few or multiple clusters per genome, as well as the occurrence of scattered copies (Little and Braaten 1989). The linkage of 5S rDNA + NTS with other types of sequences, such as other multigene families, transposable elements (TEs), and microsatellites, has also been reported (Drouin and Moniz de Sá 1995; Cross and Rebordinos 2005; Merlo et al. 2013; Anjos et al. 2015).

Among grasshoppers, the knowledge of 5S rDNA evolution was primarily obtained from chromosomal analysis. To date, cytogenetic mapping through fluorescent in situ hybridization (FISH) was performed in approximately 50 species belonging to the distinct families Acrididae (Cabralde-Mello et al. 2011a; Palacios-Gimenez et al. 2013), Romaleidae (Anjos et al. 2013; Neto et al. 2013), and Proscopiidae (Cabral-de-Mello et al. 2011b). In contrast, few studies examining the molecular organization and evolution of $5 \mathrm{~S}$ rDNA have been published. Here to contribute with the understanding of the patterns of evolution of $5 \mathrm{~S}$ rDNA among grasshoppers, we analyzed this sequence in two congeneric grasshopper species, i.e., Abracris dilecta and A. flavolineata (Acrididae: Ommatolampidinae). Two distinct types of 5S rDNA units in each species were recognized, and their nucleotide sequences were characterized and used as probes for chromosomal mapping using FISH. The results of molecular and chromosomal data are discussed to shed light on possible mechanisms involved in $5 \mathrm{~S}$ rDNA + NTS evolution.

\section{Materials and methods}

\section{Animals, chromosome obtaining, and DNA extraction}

Male and female adults from Abracris dilecta (8 individuals) were collected in Misiones, Posadas, Argentina, and 12 individuals from A. flavolineata were collected in Rio Claro, São Paulo, Brazil. The chromosomes were obtained from the testes or from the gastric cecum following the protocol described by Castillo et al. (2011). Whole animals were stored in $100 \%$ ethanol in a freezer at $-20{ }^{\circ} \mathrm{C}$ until used for DNA extraction following the protocol of Sambrook and Russel (2001).

\section{S rDNA isolation, cloning, and sequence analysis}

The 5S rDNA and the NTS region were obtained through PCR using the divergent primers 5S-NTS-F $5^{\prime}$ TACCGGTTCTCGTCCGATCAC and 5S-NTS-R 5' TACAGCGTGCTATGGCCGTTG, which was designed based on conserved region of the 5S rDNA sequence of some insects available in NCBI database. PCR was carried out using $10 \times$ PCR Rxn Buffer, $0.2 \mathrm{mM} \mathrm{MgCl} 2,0.16 \mathrm{mM}$ dNTPs, $2 \mathrm{mM}$ each primer, $1 \mathrm{U}$ of Taq Platinum DNA Polymerase (Invitrogen, San Diego, CA, USA), and 50-100 ng/ $\mu 1$ template DNA. The mixture was subjected to an initial denaturation at $94{ }^{\circ} \mathrm{C}(5 \mathrm{~min})$ and 30 cycles at $94{ }^{\circ} \mathrm{C}(30 \mathrm{~s})$, $55{ }^{\circ} \mathrm{C}(30 \mathrm{~s})$, and $72{ }^{\circ} \mathrm{C}(80 \mathrm{~s})$, with a final extension at $72{ }^{\circ} \mathrm{C}$ for $5 \mathrm{~min}$. The PCR products were separated in a $1 \%$ agarose gel, and the DNA bands were purified using the Zymoclean ${ }^{\mathrm{TM}}$ Gel DNA Recovery Kit (Zymo Research Corp., The Epigenetics Company, USA) according to the manufacturer's recommendations. The purified PCR products were ligated to the plasmid pGEM-T (Promega, Madison, WI, USA), and the recombinant constructs were used to transform DH5 $\alpha$ Escherichia coli competent cells. Positive clones were sequenced using an ABI Prism 3100 automatic DNA sequencer (Applied Biosystems, Foster City, CA, USA) with a Dynamic Terminator Cycle Sequencing Kit (Applied Biosystems).

\section{Sequence analysis}

The quality of the sequences was determined using the Geneious 4.8.5 software (Drummond et al. 2009). The consensus sequences were subjected to BLAST (Altschul et al. 1990) searches on the NCBI website (http://www.ncbi. nlm.nih.gov/blast) and, as expected, were recognized as $5 \mathrm{~S}$ rRNA genes plus NTS regions. The sequences were deposited in the NCBI database under the following accession numbers: KU958103-KU958125. For DNA sequence analyses, the basic sequence statistics were computed with the program DnaSP v.5.10.01 (Librado and Rozas 2009). Phylogenetic and molecular evolutionary relationships among sequences were inferred by neighbor-joining (NJ) using the implemented option in MEGA5 (Tamura et al. 2011) and the proportion of nucleotide differences ( $p$ distance).

\section{Fluorescent in situ hybridization}

Fluorescent in situ hybridization (FISH) was performed following the protocol proposed by Pinkel et al. (1986) 

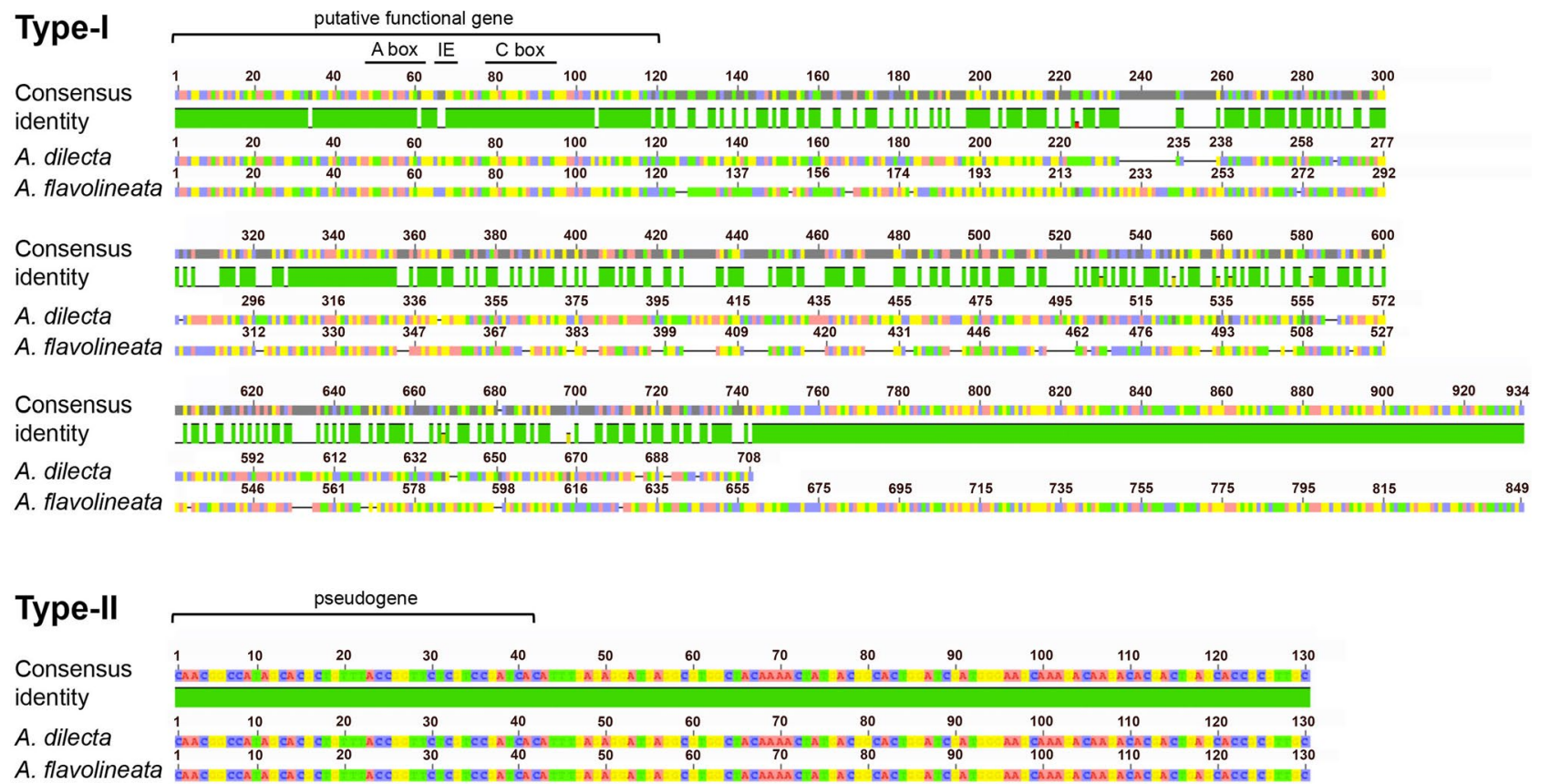

Fig. 1 Alignments of monomeric consensus units of type-I and typeII sequences of $A$. dilecta and A. flavolineata. The $5 \mathrm{~S}$ rDNA gene or pseudogene regions are indicated, and the rest of the sequence corresponds to the NTS regions. Note the difference in size and variability

with modifications by Cabral-de-Mello et al. (2010). The probes were labeled with digoxigenin-11-dUTP (Roche) or biotin-14-dATP (Invitrogen) through PCR and nick translation, respectively. Probes labeled with digoxigenin11-dUTP were detected using anti-digoxigenin-rhodamine (Roche), and probes labeled with biotin-14-dATP were detected using streptavidin and Alexa Fluor 488 conjugate (Invitrogen). The preparations were counterstained using 4', 6-diamidine-2'-phenylindole dihydrochloride (DAPI) and mounted using Vectashield (Vector, Burlingame, CA, USA). The chromosomes and FISH signals were observed using an Olympus microscope BX61 equipped with a fluorescent lamp and appropriate filters. The photographs were recorded using a DP70 cooled digital camera. The images were merged and optimized for brightness and contrast using Adobe Photoshop CS2 software.

\section{Results}

Electrophoresis of the 5S rDNA PCR amplification products revealed two fragments distinct in size for each species. The larger fragments were approximately $850 \mathrm{bp}$ for A. dilecta and $880 \mathrm{bp}$ for $A$. flavolineata, while the smaller ones were approximately $130 \mathrm{bp}$ in both species. The larger fragments were named type-I and the smaller were named type-II. These fragments will be referred to as Ad-type-I of the sequence between the species for type-I and the complete similarity for type-II. In type-I, the ICRs are also indicated. Each color corresponds to different nucleotides, blue cytosine, red adenine, yellow guanine, light green thymine

and Ad-type-II for A. dilecta, and Af-type-I and Af-typeII for A. flavolineata. The consensus sequences from a total of 23 clones, 11 from type-I (six from A. dilecta and five from A. flavolineata) and 12 from type-II (five from A. dilecta and seven from $A$. flavolineata), were obtained for molecular analysis. The comparison of the PCR fragments with the $5 \mathrm{~S}$ rDNA coding region from A. flavolineata (GenBank accession number KC936996) confirmed the isolation of the entire 5S rDNA gene unit or a portion of it. For the larger fragments, the entire 5S rDNA 120-bp gene was recognized for all clones. In contrast, the smaller fragments contained invariably only $41 \mathrm{bp}$ of the $5 \mathrm{~S}$ rDNA gene; these were considered pseudogenes. The remaining base pairs for the type-I and type-II sequences in each species were considered NTS regions (Fig. 1). The comparison between the large and small fragments confirmed that the small fragments contain only $41 \mathrm{bp}$ of the $5 \mathrm{~S}$ rDNA gene, corresponding to the initial region.

The type-I fragments contained the internal control region (ICR); i.e., the A box, the intermediate element (IE), and the $\mathrm{C}$ box. The poly- $\mathrm{T}$ motif was evident at the beginning of the NTS region for Ad-type-I and Af-type-I. These elements were not noticed in the type-II fragments (Fig. 1). The NTS regions varied between 588 and 591 bp for Ad-type-I and between 707 and 732 bp for Af-type-I. These differences were related to base deletions or insertions. For Ad-type-II and Af-type-II, the NTS were 89 bp for all clones (Table 1). 
Table 1 Polymorphism by $5 \mathrm{~S}$ rDNA unit regions in Abracris species

Fig. 2 a Schematic representation of the two 5S rDNA units observed in A. dilecta and A. flavolineata. In the NTS region of type-I units, the regions with similarity with TEs are indicated, b chromosomal mapping of $5 \mathrm{~S}$ rDNA units in metaphase I chromosomes of A. dilecta and the female mitotic metaphase of A. flavolineata. Note the single bivalent bearing the type-II and the multiple signals for type-I. Chromosomes bearing signals are indicated, and the arrowhead shows the B chromosome

\begin{tabular}{llllrrrrr}
\hline & Region & Size & $n$ & $m$ & $s$ & $h$ & $\pi$ & SD \\
\hline Type-I & & & & & & & & \\
A. dilecta & Entire unit & $713-714$ & 6 & 85 & 83 & 6 & 0.04922 & 0.00983 \\
& 5S rDNA & 120 & 6 & 9 & 9 & 3 & 0.02500 & 0.01414 \\
& NTS & $588-591$ & 6 & 76 & 74 & 6 & 0.05417 & 0.00991 \\
A. flavolineata & Entire unit & 858 & 5 & 39 & 37 & 5 & 0.02236 & 0.00379 \\
& 5S rDNA & 120 & 5 & 13 & 12 & 5 & 0.05083 & 0.01308 \\
& NTS & $707-732$ & 5 & 26 & 25 & 5 & 0.01750 & 0.00291 \\
Type-II & & & & & & & & \\
A. dilecta & Entire unit & 130 & 5 & 4 & 4 & 5 & 0.01385 & 0.00296 \\
& 5S rDNA & 41 & 5 & 1 & 1 & 2 & 0.00976 & 0.00579 \\
& NTS & 89 & 5 & 1 & 1 & 2 & 0.00833 & 0.00494 \\
A. flavolineata & Entire unit & 130 & 7 & 6 & 6 & 3 & 0.02198 & 0.00639 \\
& 5S rDNA & 41 & 7 & 0 & 0 & 1 & 0.00000 & 0.00000 \\
& NTS & 89 & 7 & 6 & 6 & 3 & 0.0321 & 0.00933 \\
\hline
\end{tabular}

$n$ number of sequences, $m$ number of mutations, $s$ number of polymorphic sites, $h$ number of haplotypes, $\pi$ nucleotide variability, $S D$ standard deviation

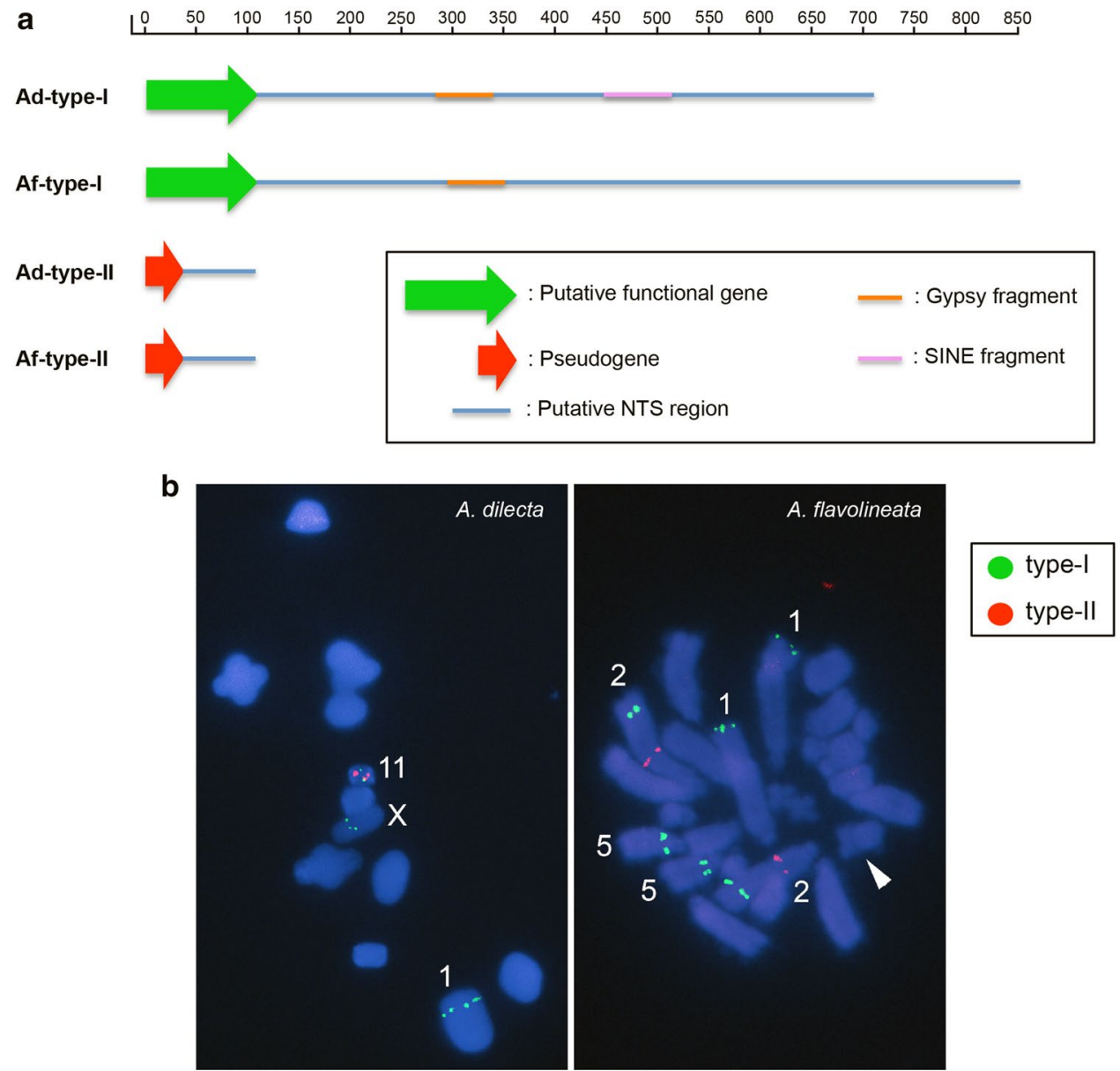

The search for similarity with other repetitive elements in RepBase (http://www.girinst.org/repbase/) using CENSOR software identified gypsy and Short Interspersed Nuclear
Element (SINE) fragments for Ad-type-I and a gypsy fragment for Af-type-I (Fig. 2a). For the Ad-type-II and Af-typeII NTS, no associated repetitive sequences were observed. 
The FISH analysis using the type-I and type-II sequences as probes in both species resulted in distinct patterns of chromosomal distribution for these elements. While the type-I was located in multiple chromosomal loci, the typeII was placed only in two chromosomes, corresponding to one bivalent. In A. dilecta, the type-I sequence was placed in chromosomes 1,11 , and X, proximally, terminally, and interstitially located, respectively. For A. flavolineata, the type-I sequence was located in pairs 1, 2, and 5, presenting terminal, interstitial, and proximal locations, corresponding to the same pattern observed through FISH using as probe only the 5S rDNA gene (Bueno et al. 2013). The type-II sequence was placed proximally in pair 11 of $A$. dilecta and interstitially in pair 2 of A. flavolineata (Fig. 2b). For A. flavolineata, no signal was recognized in the $\mathrm{B}$ chromosome.

The molecular analysis of sequence variability at the intraspecific level revealed distinct nucleotide variability depending on the species, sequence (type-I or type-II), and the specific region (5S rDNA or NTS). Considering the entire 5S rDNA unit, type-I sequences were more variable than type-II sequences, but considering the 5S rDNA and NTS separately, distinct patterns emerged as described in detail in Table 1. At the interspecific level, the NTS of typeI sequences was highly differentiated, while the NTS from type-II was remarkably conserved. The gene or pseudogene regions were similar interspecifically (Fig. 1).

\section{Discussion}

The organization of distinct classes of 5S rDNA at the molecular and/or chromosomal level is widely known in vertebrates, mainly fish (see, e.g., Martins and Galetti Jr 2001a, b; Pinhal et al. 2011; Merlo et al. 2012a, b, 2013), and to a lesser extent in other groups, such as amphibians (Rodrigues et al. 2012), crustaceans (Perina et al. 2011), and mollusks (Vierna et al. 2011). The detection of at least two distinct units of 5S rDNA, as observed in the two species studied here, was frequently reported in some fish (Martins et al. 2001), Engystomops (Rodrigues et al. 2012) and Xenopus (Harper et al. 1983), amphibians, crustaceans, mollusks, and mammals (Jensen and Frederiksen 2000; Vierna et al. 2011). According to Pinhal et al. (2011), the accumulated data from teleost fish and elasmobranches fish suggest that the presence of two distinct classes of $5 \mathrm{~S}$ rDNA is a general trend. Moreover, more 5S rDNA variants were also described in fish, up to ten in Diplodus sargus (Merlo et al. 2013), as well as in some filamentous fungi with eight variants (Rooney and Ward 2005) and Pollicipes crustacean species with seven types in a group of three species (Perina et al. 2011). A remarkable pattern is that frequently the units isolated from some species present the entire 5S rRNA gene region or a significant part, differing from Abracris species, in which one of the units lost a significant part of the 5S rRNA gene. The type-II unit putatively diverged from the ancestral sequence after duplication events, being pseudogenized due to deletion of a large gene region, and conserving only a short initial part of the 5S rRNA ancestral gene. Interestingly, the occurrence of this truncated pseudogene with a high similarity in the genome of both Abracris species suggests an origin for this sequence in the common ancestor. Pseudogenes for multigene families originating in the ancestor of two species of grasshoppers were reported for U1 snDNA in Locusta migratoria and Eyprepocnemis plorans (Anjos et al. 2015).

The occurrence of ICs that function as internal promoters and downstream T-rich regions located at the $3^{\prime}$ end of the putative coding region in the type-I sequences suggest that they may have the potential for transcription in the genomes of A. dilecta and A. flavolineata. In contrast, the short size of the type-II unit suggests that it certainly is a pseudogene sequence. Truncated sequences were also reported in fish (Martins et al. 2002), filamentous fungi (Rooney and Ward 2005), and humans (Nederby-Nielsen et al. 1993). This pattern of occurrence of pseudogenes is strong evidence of birth-and-death evolutionary processes (Rooney and Ward 2005) and has been commonly observed in stingrays (Pinhal et al. 2011), Pollicipes crustaceans (Perina et al. 2011), and razor shells (Vierna et al. 2011). In this process, the number of a given sequence in the genome could be increased by duplication. Duplicated copies accumulate divergence and deleterious mutations, causing pseudogenization. However, presumably a concerted evolutionary mechanism that led to the homogenization of copies is also operating in 5S rDNA evolution. Some authors have documented both evolutionary mechanisms acting in the same genome (Pinhal et al. 2011). Apparently, mixed evolution is applicable to the 5S rDNA of Abracris species that presents a remarkable divergence between the two 5S rDNA unit types, probably caused by a birth-anddeath mechanism. However, the sequence for each unit type shares homology, perhaps resulting from concerted evolution.

Our FISH data suggest that at least some 5S rDNA sequences from distinct units are organized in tandem, allowing the detection of evident blocks in distinct chromosomal regions, but the occurrence of dispersed repeats could not be ruled out. The distinct chromosomal location, including distinct chromosomes, for the clusters of the two types of 5S rDNA units certainly contributes to the accumulation of differences between them, and the in-tandem organization facilitates the independent homogenization process in each cluster, causing independent evolution in a concerted fashion. This was also suggested for distinct fish species in which two distinct classes of 5S rDNA were detected and evolved separately due to a different chromosomal location 
(Martins and Galetti Jr 2001a, b). Divergent organization with different $5 \mathrm{~S}$ rDNA types evolving in the same chromosome was also documented in the Cichlid fish Oreochromis niloticus (Martins et al. 2002).

It is noticeable that the type-II sequences were less variable in nucleotide diversity compared to type-I in the entire region, the 5S rRNA gene, and the NTS region. Due to the putative functionality of type-I sequences, a higher conservation was expected than for type-II, but due to a multiplicity of copies for this sequence, some variation is tolerable. In the Abracris species, two main aspects could explain this pattern: (i) type-I is much larger than type-II, allowing the accumulation of more mutations, mainly in the NTS region, but also in gene region and (ii) type-I is located in both species in multiple loci placed in distinct chromosomes that could facilitate the accumulation of differential interchromosomal mutations between them, favoring diversification.

The occurrence of gypsy and/or SINE transposable elements fragments in the NTS of type-I sequences in Abracris species could have facilitated the past dynamism, favoring the variability noticed for these sequences. Moreover, it is conceivable that it could be also responsible for the distinct chromosomal location of the clusters among the species, causing intra- and interchromosomal movement for $5 \mathrm{~S}$ rDNA repeats. However, other mechanisms could also be responsible for the 5S rDNA dispersion, such as the insertion of extrachromosomal covalently closed DNA (cccDNA) and RNA-mediated transposition (reviewed by Douin and Moniz de Sá 1995). Other cases of transposable element association with 5S rDNA units mediating sequence dynamism were documented in the fish Diplodus sargus in which ten variants of $5 \mathrm{~S}$ rDNA unit were described (Merlo et al. 2013). In the fish Gymnotus paraguensis, the multiplication of 5S rDNA to 19 chromosomal pairs was attributed to the presence of the transposable element Tc1-like in the NTS (da Silva et al. 2011).

Finally, our data reveal that the distinct organization for 5S rDNA noticed in other animal groups is also present among grasshoppers, at least in the species studied here. Moreover, the impact of chromosomal organization and association with other repetitive sequences, such as TEs, in the evolutionary history of 5S rDNA in Abracris grasshoppers is highlighted, leading to distinct fates; i.e., high variability or conservation, resulting from concerted evolution or birth-and-death. Among other grasshoppers, higher chromosomal variability for the 5S rDNA gene was noticed, with species presenting multiple clusters, including clusters in all chromosome pairs, which predicts a possible association with TEs (Cabral-de-Mello et al. 2011a), similar to Abracris species. The diversification of cluster number could be followed by higher sequence variability. Some species of grasshoppers serve as good models to test this hypothesis.
Acknowledgments The authors are grateful to the "Parque Estadual Edmundo Navarro de Andrade" administration for sample collecting authorization and to the two anonymous reviewers for valuable suggestions. This study was partially supported by the Fundação de Amparo a Pesquisa do Estado de São Paulo-FAPESP (process numbers 2011/19481-3 and 2014/11763-8) and Coordenadoria de Aperfeiçoamento de Pessoal de Nível Superior-CAPES. DB and OMPG acknowledge the scholarships obtained from FAPESP (process numbers 2011/18028-3 and 2014/02038-8, respectively). DAM was supported by Consejo Nacional de Investigaciones Científicas y Técnicas-CONICET from Argentina. DCCM was the recipient of a research productivity fellowship from the Conselho Nacional de Desenvolvimento Científico e Tecnológico-CNPq (process number 304758/2014-0).

\section{Compliance with ethical standards}

Funding This study was founded by Fundação de Amparo a Pesquisa do Estado de São Paulo-FAPESP (process numbers 2011/19481-3 and 2014/11763-8) and Coordenadoria de Aperfeiçoamento de Pessoal de Nível Superior-CAPES.

Conflict of interest Danilo Bueno declares that he has no conflict of interest. Octavio Manuel Palacios-Gimenez declares that he has no conflict of interest. Dardo Andrea Martí declares that he has no conflict of interest. Tatiane Casagrande Mariguela declares that she has no conflict of interest. Diogo Cavalcanti Cabral-de-Mello declares that he has no conflict of interest.

Ethical approval All applicable international, national, and/or institutional guidelines for the care and use of animals were followed.

\section{References}

Altschul SF, Gish W, Miller W, Myers EW, Lipman DJ (1990) Basic local alignment search tool. J Mol Biol 215:403-410

Anjos A, Loreto V, de Souza MJ (2013) Chromosome mapping of ribosomal genes and histone $\mathrm{H} 4$ in the genus Radacridium (Romaleidae). Genet Mol Biol 36:336-340

Anjos A, Ruiz-Ruano FJ, Camacho JPM, Loreto V, Cabrero J, de Souza MJ, Cabral-de-Mello DC (2015) U1 snDNA clusters in grasshoppers: chromosomal dynamics and genomic organization. Heredity 114:207-219

Bueno D, Palacios-Gimenez OM, Cabral-de-Mello DC (2013) Chromosomal mapping of repetitive DNAs in Abracris flavolineata reveal possible ancestry for the B chromosome and surprisingly H3 histone spreading. PLoS ONE 8:e66532

Cabral-de-Mello DC, Moura RC, Martins C (2010) Chromosomal mapping of repetitive DNAs in the beetle Dichotomius geminatus provides the first evidence for an association of 5S rRNA and histone $\mathrm{H} 3$ genes in insects, and repetitive DNA similarity between the B chromosome and A complement. Heredity 104:393-400

Cabral-de-Mello DC, Cabrero J, López-León MD, Camacho JPM (2011a) Evolutionary dynamics of 5S rDNA location in acridid grasshoppers and its relationship with $\mathrm{H} 3$ histone gene and $45 \mathrm{~S}$ rDNA location. Genetica 139:921-931

Cabral-de-Mello DC, Martins C, Souza MJ, Moura RC (2011b) Cytogenetic mapping of $5 \mathrm{~S}$ and $18 \mathrm{~S}$ rRNAs and $\mathrm{H} 3$ histone genes in 4 ancient Proscopiidae grasshopper species: contribution to understanding the evolutionary dynamics of multigene families. Cytogenet Genome Res 132:89-93

Castillo ER, Taffarel A, Marti DA (2011) Una técnica alternativa para el cariotipado mitótico en saltamontes: bandeo C y Fluorescente 
en Adimantus ornatissimus (Orthoptera: Acrididae). Rev Cienc Tecnol 16:30-34

Cross I, Rebordinos L (2005) 5S rDNA and U2 snRNA are linked in the genome of Crassostrea angulate and Crassostrea gigas oysters: does the $(\mathrm{CT})_{n} \cdot(\mathrm{GA})_{n}$ microsatellite stabilize this novel linkage of large tandem arrays? Genome 48:1116-1119

da Silva M, Matoso DA, Vicari MR, de Almeida MC, Margarido VP, Artoni RF (2011) Physical mapping of 5S rDNA in two species of knifefishes: Gymnotus pantanal and Gymnotus paraguensis (Gymnotiformes). Cytogenet Genome Res 134:303-307

Drouin G, Moniz de Sá M (1995) The concerted evolution of 5S ribosomal genes linked to the repeat units of other multigene families. Mol Biol Evol 12:481-493

Drummond AJ, Ashton B, Cheung M, Heled J, Kearse M, Moir R et al (2009) Geneious v4.8.5. http://www.geneious.com

Haper ME, Price J, Korn LJ (1983) Chromosomal mapping of Xenopus 5S genes: somatic-type versus oocyte-type. Nucl Acids Res 11:2313-2323

Jensen LR, Frederiksen S (2000) The 5S rRNA genes in Macaca fascicularis are organized in two large tandem repeats. Biochim Biophys Acta 1492:537-542

Librado P, Rozas J (2009) DnaSP v5: a software for comprehensive analysis of DNA polymorphism data. Bioinformatics 25:1451-1452

Little RD, Braaten DC (1989) Genomic organization of human 5S rDNA and sequence of one tandem repeat. Genomics 4:376-383

Long EO, Dawid IB (1980) Repeated genes in eukaryotes. Ann Rev Biochem 49:727-764

Martins C, Galetti PM Jr (2001) Organization of 5S rDNA in species of the fish Leporinus: two different genomic locations are characterized by distinct nontranscribed spacers. Genome 44:903-910

Martins C, Galleti PM Jr (2001) Two 5S rDNA arrays in neotropical fish species: is it a general rule for fishes? Genetica 111:439-446

Martins C, Wasko AP, Oliveira C, Porto-Foresti F, Parise-Maltempi PP, Wright JM, Foresti F (2002) Dynamics of 5S rDNA in the tilapia (Oreochromis niloticus) genome: repeat units, inverted sequences, pseudogenes and chromosome loci. Cytogenet Genome Res 98:78-85

Merlo MA, Cross I, Palazón JL, Úbeda-Manzanaro M, Sarasquete C, Rebordinos L (2012a) Evidence for 5S rDNA horizontal transfer in the toadfish Halobatrachus didactylus (Schneider, 1801) based on the analysis of three multigene families. BMC Evol Biol 12:201

Merlo MA, Pacchiarini T, Portela-Bens S, Cross I, Manchado M, Rebordinos L (2012b) Genetic characterization of Plectorhinchus mediterraneus yields important clues about genome organization and evolution of multigene families. BMC Genet $13: 33$

Merlo MA, Cross I, Manchado M, Cárdenas S, Rebordinos L (2013) The 5S rDNA high dynamism in Diplodus sargus is a transposon-mediated mechanism. Comparison with other multigene families and Sparidae species. J Mol Evol 76:83-97

Nederby-Nielsen J, Hallenberg C, Frederiksen S, Sorensen PD, Lomholt B (1993) Transcription of human 5S rRNA genes is influenced by an upstream DNA sequence. Nucleic Acids Res 21:3631-3636
Nei M, Rooney AP (2005) Concerted and birth-and-death evolution of multigene families. Ann Rev Genetics 39:121-152

Neto MSR, de Souza MJ, Loreto V (2013) Chromosomal evolution of rDNA and $\mathrm{H} 3$ histone genes in representative Romaleidae grasshoppers from northeast Brazil. Mol Cytogenetics 6:41

Palacios-Gimenez OM, Castillo ER, Martí DA, Cabral-de-Mello DC (2013) Tracking the evolution of sex chromosome systems in Melanoplinae grasshoppers through chromosomal mapping of repetitive DNA sequences. BMC Evol Biol 13:167

Perina A, Seoane D, González-Tizón AM, Rodríguez-Fariña F, Martínez-Lage A (2011) Molecular organization and phylogenetic analysis of $5 \mathrm{~S}$ rDNA in crustaceans of the genus Pollicipes reveal birth-and-death evolution and strong purifying selection. BMC Evol Biol 11:304

Pinhal D, Yoshimura TS, Araki CS, Martins C (2011) The 5S rDNA family evolves through concerted and birth-and-death evolution in fish genomes: an example from freshwater stingrays. BMC Evol Biol 11:151

Pinkel D, Straume T, Gray JW (1986) Cytogenetic analysis using quantitative, high-sensitivity, fluorescence hybridization. Proc Natl Acad Sci USA 83:2934-2938

Rebordinos L, Cross I, Merlo A (2013) High evolutionary dynamism in 5S rDNA of fish: State of the Art. Cytogenet Genome Res 141:103-113

Rodrigues DS, Rivera M, Lourenço LB (2012) Molecular organization and chromosomal localization of 5S rDNA in Amazonian Engystomops (Anura, Leiuperidae). BMC Genet 13:17

Rooney AP, Ward TJ (2005) Evolution of a large ribosomal RNA multigene family in filamentous fungi: birth and death of a concerted evolution paradigm. Proc Natl Acad Sci USA 102:5084-5089

Sambrook J, Russel DW (2001) Molecular cloning. In: A laboratory manual. 3rd edn. Cold Spring Harbor Laboratory Press, New York

Tamura K, Peterson D, Peterson N, Stecher G, Nei M, Kumar S (2011) MEGA5: molecular evolutionary genetics analysis using maximum likelihood evolutionary distance, and maximum parsimony methods. Mol Biol Evol 28:2731-2739

Úbeda-Manzanaro M, Merlo MA, Palazón JL, Cross I, Sarasquete C, Rebordinos L (2010) Chromosomal mapping of the major and minor ribosomal genes, (GATA) $n$ and U2 snRNA gene by double-colour FISH in species of the Batrachoididae family. Genetica 138:787-794

Vierna J, Jensen KT, Martínez-Lage A, González-Tizón AM (2011) The linked units of 5S rDNA and U1 snDNA of razor shells (Mollusca: Bivalvia: Pharidae). Heredity 107:127-142

Vierna J, Wehner S, Zu Siederdissen CH, Martínez-Lage A, Marz M (2013) Systematic analysis and evolution of 5S ribosomal DNA in metazoans. Heredity 111:410-421

Vizoso M, Vierna J, González-Tizón AM, Martínez-Lage A (2011) The 5S rDNA gene family in Mollusks: characterization of transcriptional regulatory regions, prediction of secondary structures, and long-term evolution, with special attention to Mytilidae mussels. J Heredity 102:433-447

Wasko PA, Martins C, Wright JM, Galetti PM Jr (2001) Molecular organization of $5 \mathrm{~S}$ rDNA in fishes of the genus Brycon. Genome 44:893-902 